\title{
SOME INTRICATE NONINVERTIBLE LINKS
}

\author{
BY WILBUR WHITTEN
}

Communicated by N. E. Steenrod, April 15, 1970

Let $L$ be an oriented, ordered link imbedded in the oriented 3 sphere $S^{3}$, and let $\mu$ and $\kappa$ be integers such that $1 \leqq \kappa<\mu$. We say that $L$ is a generalized noninvertible link for the pair $\mu, \kappa$ (or a $(\mu, \kappa) I$ link) if it satisfies:

(i) L has $\mu$ components;

(ii) Each sublink with $\kappa$ or fewer components is invertible;

(iii) Each sublink with more than $\kappa$ components is noninvertible.

$L$ is invertible provided it is of the same (oriented) type as its inverse. The inverse of $L$ is obtained by reversing the orientation of each component of $L$.

Now $(2,1) I$ links were exhibited in $[2]$ and a $(\mu, \mu-1) I$ link was given in [3] for each $\mu \geqq 3$. In this announcement we outline the construction of a generalized noninvertible link for each pair $\mu, \kappa$ such that $1 \leqq \kappa<\mu$ and $\mu \geqq 3$. Details will appear elsewhere.

1. Two propositions. The following propositions clear the way for the constructive type proof of the main Theorem 2.1. An induction argument together with results of [2] yields a proof of

Proposition 1.1. For each integer $\mu \geqq 2$, there exists $a(\mu, 1) I$ link in $S^{3}$.

The combined contents of [2] and [3] are stated in

Proposition 1.2. For each integer $\mu \geqq 2$, there exists $a(\mu, \mu-1) I$ link in $S^{3}$.

2. $(\mu, \kappa) I$ links. The main result is

Theorem 2.1. For each pair of integers $\mu, \kappa$ such that $1 \leqq \kappa<\mu$, there is a generalized noninvertible link $\&$ in $S^{3}$ satisfying (i), (ii), and (iii) of the introduction.

Outline of construction. By Propositions 1.1 and 1.2, we need consider only those integers $\mu, \kappa$ for which $2 \leqq \kappa<\mu-1$. We relax this, however, and assume only that $2 \leqq \kappa<\mu$.

Set $\nu=\left(\begin{array}{c}\mu-1 \\ \kappa\end{array}\right)$. Let $Q_{1}, \ldots, Q_{\nu}$ be a collection of disjoint 3-cells in $S^{3}$

AMS 1970 subject classifications. Primary 55A25; Secondary 55A10.

Key words and phrases. Classical knot theory, noninvertible knots and links. 
each of which is in the shape of a solid cylinder. In each $Q_{l}, \quad(l=1$, $\ldots, \nu)$, construct the oriented, ordered link

$$
L_{l}=(l, 1) \cup \cdots \cup\left(l, \alpha_{l 2}-1\right) \cup\left(l, \alpha_{l 2}\right) \cup \cdots \cup\left(l, \alpha_{l_{k+1}}\right)
$$

as shown in Figure 1. (Two small arcs of each component are to lie on $\partial Q_{l}$ as indicated with the remainder of $L_{l}$ in Int $Q_{l}$ ) The set $\left\{\alpha_{l 2}, \ldots, \alpha_{l k+1}\right\}$ is the $l$ th combination of the integers $2, \ldots, \mu$ taken $\kappa$ at a time, and in the lexicographical ordering of these combinations.

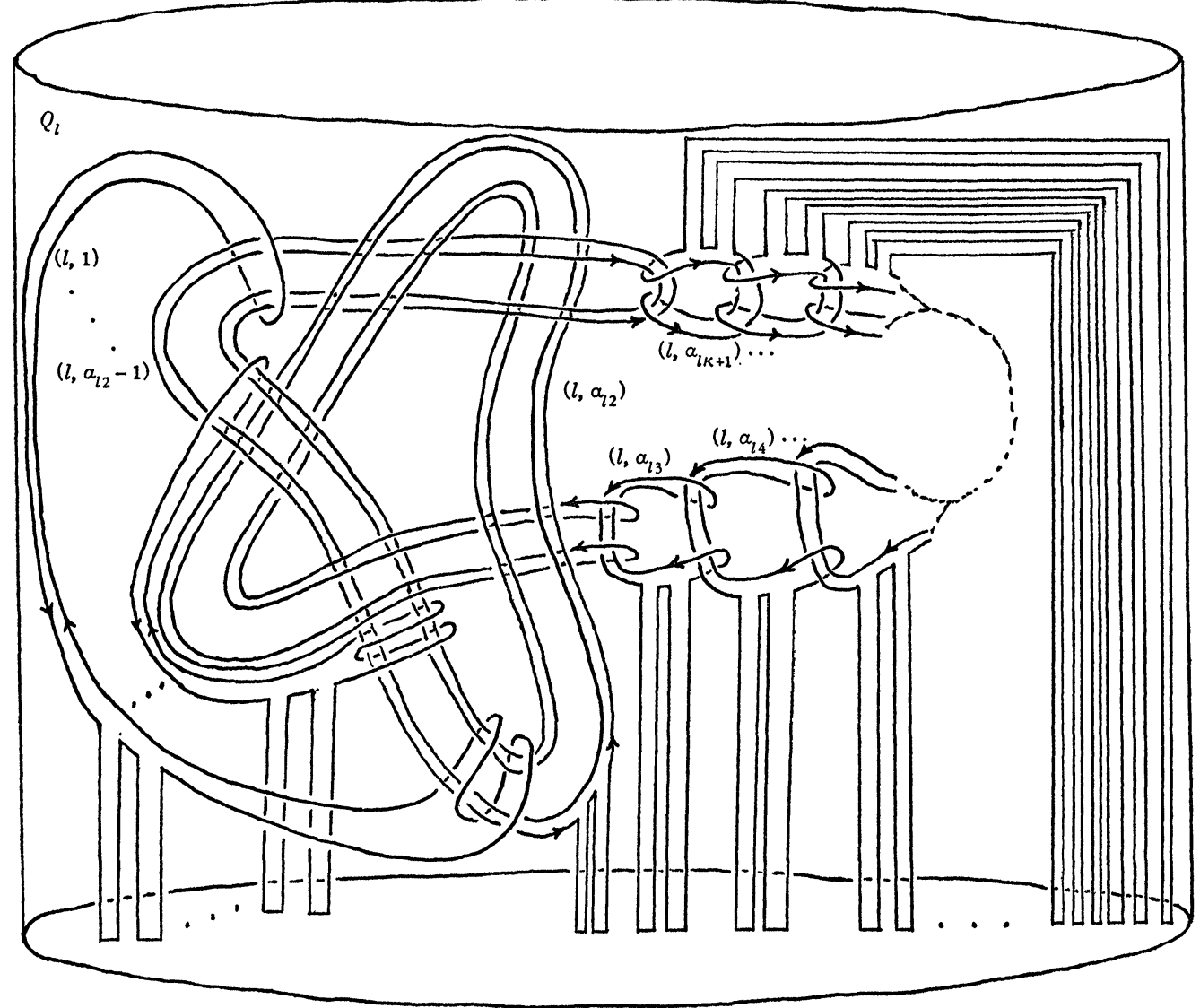

FIGURE 1

Now let $\left(l_{1}, \alpha\right), \ldots,\left(l_{t(\alpha)}, \alpha\right)$ be the collection of all those pairs whose second coordinate is $\alpha$. We assume that $l_{1}<\ldots<l_{t(\alpha)}$, set $\mathfrak{K}_{\alpha}=\left(l_{1}, \alpha\right) \# \ldots \#\left(l_{t(\alpha)}, \alpha\right)$, and $\mathfrak{L}=\mathfrak{K}_{1} \cup \ldots \cup \mathfrak{K}_{\mu}$. (R. H. Fox 
gives a nice account of the composition operation \# in $\S 7$ of [1].) The compositions, formed inductively with respect to $\alpha$, are to be made by running two parallel arcs (each with proper orientation) in the obvious nice way from $\left(l_{m}, \alpha\right)$ to $\left(l_{m+1}, \alpha\right),(m=1, \ldots, t(\alpha)-1)$, and then deleting the appropriate small arcs on $\partial Q_{l_{m}}$ and $\partial Q_{l_{m+1}}$. Several routine requirements on the placements of these pairs of arcs are also made.

That $\mathscr{L}$ is a $(\mu, \kappa) I$ link follows from the construction and the following properties of $L_{l}$ in Figure 1:

1. For each $j=1, \ldots, \alpha_{l 2}-1$, the sublink $(l, j) \cup\left(l, \alpha_{l 2}\right) \cup \ldots \cup$ $\left(l, \alpha_{l \kappa+1}\right)$ is a $(\kappa+1, \kappa) I$ link. Methods similar to those of [3] prove this.

2. Any sublink of $L_{l}$ which is obtained by removal of one of the components $\left(l, \alpha_{l 2}\right), \ldots,\left(l, \alpha_{l} \kappa_{+1}\right)$ is completely splittable.

\section{REFERENCES}

1. R. H. Fox, A quick trip through knot theory, Topology of 3-manifolds and Related Topics (Proc. Univ. of Georgia Inst., 1961) Prentice-Hall, Englewood Cliffs, N. J., 1962, pp. 120-167. MR 25 \#3522.

2. W. Whitten, A pair of non-invertible links, Duke Math. J. 36 (1969), 695-698.

3. - On noninvertible links with invertible proper sublinks, Proc. Amer. Math. Soc. (to appear).

University of Southwestern Louisiana, Lafayette, Louisiana 70501 